\title{
Partial replication of a DRD4 association in ADHD individuals using a statistically derived quantitative trait for ADHD in a family-based association test
}

2007 Biological Psychiatry, 62, 985-990 - doi:10.1016/j.biopsych.2007.03.006

Jessica Lasky-Su ${ }^{1}$, Tobias Banaschewski ${ }^{4}$, Jan Buitelaar ${ }^{6}$, Barbara Franke, Keeley Brookes $^{2}$, Edmund Sonuga-Barke ${ }^{9}$, Richard Ebstein ${ }^{12}$, Jacques Eisenberg ${ }^{11}$, Michael Gill ${ }^{13}$, Iris Manor , Ana Miranda , Fernando Mulas, Robert D. Oades ${ }^{6}$, Herbert Roeyers ${ }^{6}{ }^{6}$, Aribert Rothenberger, Joseph Sergeant ${ }^{4}$, Hans-Christoph Steinhausen ${ }^{15}$, Eric Taylor ${ }^{3}$, Kaixin Zhou ${ }^{2}$, Margaret Thompson ${ }^{9}$, Philip Asherson , Stephen V Faraone ${ }^{1,17^{*}}$

1

Department of Psychiatry, SUNY Upstate Medical University, Syracuse, NY, United States MRC Social Genetic Developmental and Psychiatry Centre, Institute of Psychiatry, London, United Kingdom University Clinic for Child and Adolescent Psychiatry, Essen, Germany Geha MHC, Petach-Tikva, Israel Child and Adolescent Psychiatry, University of Göttingen, Germany Department of Psychiatry, Radboud University Nijmegen Medical Center, Nijmegen, The Netherlands Department of Developmental and Educational Psychology, University of Valencia, Spain Triversum, Kees Boekestraat 5, 1817 EZ Alkmaar, The Netherlands ${ }^{9}$ Amsterdam Medical Centre - de Bascule, Amsterdam, The Kees Boekestraat 5, 1817 EZ Alkmaar, The Netherlands Amsterdam Medical Centre - de Bascule, Amsterdam, The Netherlands School of Psychology, University of Southampton, Highfield, Southampton, United Kingdom Vrije Universiteit, De Boelelaan 11051081 HV, Amsterdam, The Netherlands ${ }^{12}$ ADHD Clinic, Geha Mental Health Center, Petak Tikvah, Israel $^{13}$ S. Herzog Memorial Hospital, Jerusalem, Israel Department of Psychiatry, Trinity Centre for Petak Tikvah, Israel S. Herzog Memorial Hospital, Jerusalem, Israel Department of Psychiatry, Trinity Centre for Health Sciences, St. James's Hospital, Dublin, Ireland Ghent University, Dunantlaan 2, B-9000 Ghent, Belgium Department of Child and Adolescent Psychiatry, University of Zurich, Switzerland Department of Neuroscience \& Physiology, SUNY Upstate Medical University ${ }^{17}$

Corresponding Author: Stephen V. Faraone, Ph.D.; Department of Psychiatry; SUNY Upstate Medical University; 750 East Adams Street; Syracuse, NY 13210; Phone: 315-464-3113; Fax: 315-849-1839; E-mail: faraones@upstate.edu

\section{ABSTRACT}

\section{Background:}

Previous research found an association between SNPs in the promoter region of DRD4 and statistically-derived phenotypes generated from ADHD symptoms. We sought to replicate this finding by using the same methodology in an independent sample of ADHD individuals.

\section{Methods:}

Four SNPs were genotyped in and around DRD4 in 2,631 individuals in 642 families. We developed a quantitative phenotype at each SNP by weighting nine inattentive and nine hyperactive-impulsive symptoms. The weights were selected to maximize the heritability at each SNP. Once a quantitative phenotype was generated at each SNP, the screening procedure implemented in PBAT was used to select and test the five SNPs / genetic model combinations with the greatest power to detect an association within each candidate gene. 
Results:

One of the four SNPs was associated with the quantitative phenotypes generated from the ADHD symptoms (corrected p-values $=0.02$ ). A rank ordering of the correlation between each of the ADHD symptoms and the quantitative phenotype suggested that hyperactive-impulsive symptoms were more strongly correlated with the phenotype; however, including inattentive symptoms was necessary to achieve a significant result.

\section{Conclusions:}

This study partially replicated a previous finding by identifying an association between rs7124601 and a quantitative trait generated from ADHD symptoms. Rs7124601 is LD with the SNPs identified previously. In contrast to the previous study, this finding suggests that both hyperactive-impulsive and inattentive symptoms are important in the association.

\section{INTRODUCTION}

Attention deficit hyperactivity disorder is characterized by symptoms of inattention and hyperactive-impulsive behavior (American Psychiatric Association, 1994). Family, twin and adoption studies have established that ADHD is a complex disorder with both genetic and environmental components (Doyle et al. 2001; Morrison and Stewart, 1971, 1973; Cantwell, 1972, 1975; Faraone, et al. 1994; Welner et al. 1977; Lopez, 1965). Heritability estimate for ADHD are now suggested to be around 76 percent (Faraone et al. 2005).

Despite the large number of studies, identifying genes associated with complex diseases has been more difficult that initially anticipated. Such difficulty has compelled researchers to develop innovative strategies to identify genes influencing complex diseases. One such strategy is phenotype refinement. Generating heritable quantitative phenotypes that more specifically describe the disorder, or characteristics of the disorder, may be usefully in elucidating our understanding of the genetics underlying these diseases. This approach has been advocated for in ADHD genetics research (Todd, 2000).

The D4 dopamine receptor gene (DRD4) is one of the best-studied genes believed to be associated with ADHD. This is primarily because of the role that DRD4 mRNA plays on the clinical characteristics of ADHD, specifically in regard to cognitive and emotional functioning (Paterson, 1999). In addition, meta-analyses of a well-known 7repeat allele in this gene were associated with ADHD (Faraone et al. 2001, 2005). Additional studies have also used quantitative phenotypes from neuropsychological testing and attention data to find (Langley et al. 2004; Manor et al. 2002) significant associations with the DRD4 7repeat allele.

Lasky-Su et al. (Lasky-Su, Biological Psychiatry in press) generated a heritable trait using ADHD symptoms and the FBAT-PC approach that is implemented in the PBAT computer program (Lange et al. 2004a). This approach has been successful in identifying disease susceptibility loci for disease such as obesity with consistent replication (Herbert et al. 2006). FBAT-PC maximizes the genetic information when multiple phenotypes are tested (Lange et al. 2004b) by using principal components analysis to construct an overall phenotype that amplifies the trait heritability by aggregating the genetic components of all measurements into a single univariate phenotype. Using this methodology, a weighting was generated for each of 18 ADHD symptoms at each SNP. Lasky-Su et al. found a significant association with the phenotypes at two adjacent SNPs in 
the promoter region of DRD4, hCV26775267 and hCV26775266 (corrected p-values = 0.012 for both SNPs) (Lasky-Su, Biological Psychiatry in press). A FBAT-PC analysis of the haplotypes in the haplotype block generated from these SNPs also resulted in a significant association. Subsequent analyses suggested that this association was driven more by the inattentive symptoms than the hyperactive-impulsive symptoms. In this study we sought to replicate these findings using an independent sample.

\section{METHODS}

\section{Subjects and sample collection}

The sample used in this research is a part of the International Multi-center Genetics project (IMAGE). Details of the subjects and sample collection for this study can found elsewhere (Brookes et al. 2006). Briefly, European Caucasian subjects were recruited from twelve specialist clinics in eight countries: Belgium, Germany, Holland, Ireland, Israel, Spain, Switzerland and United Kingdom. Ethical approval for this study was obtained from National Institute of Health registered ethical review boards for each center. Probands and their siblings were required to be between the ages of 5 and 17 at the time of entry. Probands were required to have clinical diagnosis of DSMIV combined ADHD subtype and have at least one full sibling available for ascertainment of clinical information and DNA collection. Exclusion criteria for either the probands or their siblings included autism, epilepsy, low IQ $(<70)$, brain disorders and any genetic or medical disorder associated with externalizing behaviors that might mimic ADHD. Probands were also excluded if the last medication free period was more than 2 years ago. The clinical assessments for children were made during medication-free periods, or recall back to medication-free periods. To insure that the recall was accurate, affected individuals were required to have had a medication-free period within the last two years.

The final IMAGE dataset had 776 DSM-IV combined type affected individuals, of which 674 were probands. DNA was available for both parents in 614 families $(90 \%)$ and from one parent in 49 families (7\%). To be informative in this analysis, families must have at least two genotyped people and one individual with ADHD symptom information. A summary of the sample that met these criteria is included in table 1.

\section{Clinical Measures}

Rating scales used to quantify ADHD symptoms included the Long Version of Conners' Parent Rating Scale (CPRS$\mathrm{R}: \mathrm{L})(C o n n e r s, 1996)$. This is a reliable, easyto-administer instrument that assesses the DSM-IV symptoms of ADHD in children and adolescents. The CPRS-RL was administered by investigators at each center to the parents of the affected child. There was centralized training for all who administered the CPRS-RL and the responses to questions were standardized. The symptoms described by parents were during medication-free periods, whether current or within the past two years. The inattentive and hyperactive-impulsive symptoms included are: 1 ) inability to pay attention to details; 2) difficulty with sustained attention in tasks or play activities; 3) listening problems; 4) difficulty following instructions; 5) problems organizing tasks and activities; 6) avoidance or dislike of tasks that require mental effort; 7) tendency to lose things like toys, notebooks, or homework; 8) distractibility; and 9) forgetfulness in daily activities, 10) fidgeting or squirming; 11) difficulty remaining seated; 12) restlessness; 13) difficulty playing quietly; 14) always seeming to be "on the go"; 15) excessive talking; 16) blurting out answers before hearing the full question; 17) difficulty 
waiting for a turn or in line; and 18) problems with interrupting or intruding. For each symptom, individuals were given a score ranging between 0 and 3 , where 0 indicated that the behavior was not characteristic of the individual and exhibited seldom and 3 indicated that the behavior was very characteristic of the individual was exhibited very frequently.

\section{DNA and SNP collection}

The DNA sample used in this research is a part of a larger genetic sample from the IMAGE study. Details of the DNA and SNP collection can be found elsewhere (Brookes et al. 2006). Briefly, blood samples were sent to Rutgers University Cell and DNA repository, New Jersey where DNA was extracted. In a limited number of cases where individuals were not able to supply a blood sample, DNA was extracted from a mouth swab sample at the SGDP laboratories in London (Freeman et al. 2003). DNA stocks were collated in London where they were stored, organized and plated out for further analysis. Geneservice Ltd. Cambridge (UK) performed whole genome amplification on all samples with less than $100 \mu \mathrm{g}$ stock DNA, using the REPLIg kit (Quiagen Ltd., Crawley, UK). DNA samples were arrayed into 96-well plates at a concentration of $50 \mathrm{ng} / \mu \mathrm{l}$ and delivered to Illumina Inc. (San Diego, US) under dry ice. Four SNPs were genotyped throughout DRD4, including rs3758653, rs916457, rs752306, and rs7124601. These SNPs were selected as tag SNPs to explain the LD structure throughout the gene. Each SNP was evaluated to ensure Hardy-Weinberg equilibrium ( $\alpha=0.01)$.

The initial study was genotyped prior to the HAPMAP and used Celera SNPs. These SNPs were not tag SNPs and the RS numbers for these SNPs were not clear. The attempt to replicate this finding using the IMAGE sample was performed using genotypes completed on an existing
Illumina array and therefore the replication sample was one of convenience. For these reasons the SNPs selected for replication were not the same SNPs that were used in the initial study, which limits the extent that the initial finding can be replicated directly; however, the SNPs do cover the same haplotype block structure as the previous sample.

\section{Data Cleaning}

Data cleaning procedures were performed previously on the entire IMAGE sample. Details of the cleaning procedure can be found elsewhere (Brookes et al. 2006). Briefly, PEDCHECK (O'Connell and Weeks, 1998) was run to detect single point Mendelian inconsistencies on the sample of 987 polymorphic autosomal markers in the initial dataset of 680 families and 47 families were identified with potential pedigree errors. Within these families the number of Mendelian errors ranged from 16 to 312 and could not be explained by genotyping errors. Additional analyses identified 16 DNA swaps, 8 non-paternity and 7 half-sib cases, which cleared up the Mendelian errors from 40 families. This left 7 families with unknown pedigree errors that we removed from the analysis. The final dataset for association analysis included 673 families that contained 156 sporadic Mendelian errors from 987 autosomal markers. The overall detection rate of Mendelian errors is therefore $0.02 \%$, which is consistent with the estimated efficiency of SNP markers (13 -75\%, (Douglas et al. 2002)) to detect such errors by identification of Mendelian errors and an estimated overall genotype error rate less than $0.065 \%$. The large number of pedigree errors may be explained by the complicated procedures inherent within a large international cooperative process. 
Family-Based Association Test-Principal Components (FBAT-PC)

Family-based association tests are those studies that use genetic data from family members to evaluate the possible association of a disease phenotype and a gene allele. FBAT-PC is an approach designed to aggregate the genetic information from many phenotypes into an overall phenotype that is then used in the family-based association analysis (Lange et al. 2004b); it has been implemented in the PBAT computer program (Lange et al. 2004a). The FBAT-PC methodology has been successful in identifying genetic associations with obesity that were subsequently replicated in a broad range of populations (Herbert, 2006).

Details of the FBAT-PC methodology can be found elsewhere (Lange et al. 2004b). Briefly, Lange et al. use an approach based on the conditional mean model whereby genetic effect sizes can be calculated for each phenotype in a way that is statistically independent of the genetic data that is used in the subsequent association test. These genetic effect size estimates are used along with information on the mode of inheritance and allele frequencies to estimate a matrix of genetic variances. The phenotypic variance matrix is calculated using the original set of phenotypes as well as any other desired covariates. Once the genetic and phenotypic variance matrices are generated, heritability estimates can be calculated by dividing the genetic variances by the total variance (the sum of the genetic and phenotypic matrices). The heritability estimate can change by selecting various weighting schemes for each of the phenotypes. For example, a higher heritability estimate would result when the phenotypes that have a large genetic effect are weighted more than the phenotypes with a small genetic effect. FBAT-PC selects the set of weights that generate the largest heritability possible for the selected phenotype at a given SNP. These weights are found through the use of a generalized version of principal components analysis. With these weights, the final phenotype that is used in the association analysis is calculated. We apply this methodology to 9 inattentive and 9 hyperactive-impulsive symptoms for ADHD.

In all PBAT analyses, a screening procedure is employed to limit the number of statistical tests that are performed. Details on the screening procedure can be found elsewhere (Lange et al. 2003); however a brief summary of the SNP selection is provided. First, power to detect an association with the generated univariate phenotype is calculated using the conditional mean model. Second, the subset of SNP/genetic model combinations is selected based on the power to detect a genetic association. Third, the FBAT-PC statistic is calculated for the selected SNP/ genetic model combinations and their associated phenotypes. In this analysis, the additive, dominant, and recessive genetic models were used in the screening procedure and at least 20 informative families were required for any given SNP to be screened. The $5 \mathrm{SNP} /$ genetic model combinations with the greatest power were retained and the FBAT-PC statistic was calculated. These 5 SNPs were subsequently adjusted for multiple comparisons using the false discovery rate (Benjamini and Hochberg, 1995).

The relationship between each of the ADHD symptoms and the final phenotype generated using the FBAT-PC methodology was determined by looking at the correlation between the two variables. We then ranked these correlations from the highest to the lowest to determine what symptoms most strongly contributed to the generated phenotype. 


\begin{tabular}{|c|c|c|c|}
\hline \multicolumn{4}{|c|}{$\begin{array}{l}\text { Table 1: } \\
\text { Descriptive Statistics on Individuals } \\
\text { used in the FBAT-PC Analysis }\end{array}$} \\
\hline Number of people & \multicolumn{3}{|c|}{2631} \\
\hline Number of families & \multicolumn{3}{|l|}{642} \\
\hline $\begin{array}{c}\text { Genotyped individuals } \\
\text { within a family } \\
2 \\
3 \\
4 \\
5 \\
6\end{array}$ & \multicolumn{3}{|l|}{$\begin{array}{l}9 \\
63 \\
442 \\
112 \\
16\end{array}$} \\
\hline $\begin{array}{l}\text { Gender distribution over } \\
\text { entire sample } \\
\text { Male (percent) } \\
\text { Female } \\
\text { (percent) } \\
\end{array}$ & \multicolumn{3}{|c|}{$\begin{array}{l}1546(58.8) \\
1085(41.2)\end{array}$} \\
\hline Number of Children & \multicolumn{3}{|c|}{1439} \\
\hline $\begin{array}{l}\text { Genotyped siblings within } \\
\text { a family } \\
1 \\
2 \\
3 \\
4\end{array}$ & \multicolumn{3}{|l|}{$\begin{array}{l}20 \\
469 \\
131 \\
22 \\
\end{array}$} \\
\hline $\begin{array}{l}\text { Gender distribution among } \\
\text { children } \\
\\
\text { Male } \\
\text { Female } \\
\end{array}$ & \multicolumn{3}{|c|}{$\begin{array}{l}979(68.0) \\
460(32.0) \\
\end{array}$} \\
\hline $\begin{array}{l}\text { Average a among children } \\
\text { (standard deviation) }\end{array}$ & \multicolumn{3}{|c|}{$7.92(3.09)$} \\
\hline Comorbidities & Yes & No & $\begin{array}{l}\text { Miss } \\
\text { ing }\end{array}$ \\
\hline Autism & 7 & 474 & 958 \\
\hline Conduct Disorder & 186 & 581 & 672 \\
\hline $\begin{array}{r}\text { Oppositional Defiant } \\
\text { Disorder }\end{array}$ & 488 & 280 & 671 \\
\hline Schizophrenia & 6 & 763 & 670 \\
\hline $\begin{array}{l}\text { Reactive Attachment } \\
\text { Disorder }\end{array}$ & 2 & 761 & 676 \\
\hline $\begin{array}{c}\text { Obsessive Compulsive } \\
\text { Disorder }\end{array}$ & 26 & 732 & 681 \\
\hline Substance Misuse & 13 & 755 & 671 \\
\hline Tourette's Syndrome & 15 & 754 & 670 \\
\hline Anxiety Disorder & 337 & 423 & 679 \\
\hline Bipolar Affective Disorder & 2 & 766 & 671 \\
\hline Mood Disorder & 123 & 641 & 675 \\
\hline
\end{tabular}

\section{RESULTS}

\section{Descriptive Statistics}

The summary of the sample characteristics is given in table 1.

\section{FBAT-PC Analysis}

Table 2 shows the 5 SNP/genetic model combinations selected through the screening procedure listed from highest to lowest power. Also presented are the number of informative families at each SNP, the genetic model, and the FBAT-PC p- value. Rs7124601 (physical location: 629273) achieves overall significance after adjusting for the five tests. The overtransmitted allele for rs7124601 was A. This SNP is located in intron 1 of DRD4 (http://snpper.chip.org/bio), $160 \mathrm{bp}$ from exon 2. The rank-ordered correlations between each ADHD symptom and the overall phenotypes are listed in figure 1 . The average correlation of the hyperactiveimpulsive symptoms was 0.16 while the average correlation for the inattentive symptoms was 0.02 . The top three most influential variables on the phenotype were all hyperactive-impulsive variables including 1) often blurts out answers before questions have been completed, 2) Is often "on the go" or often acts as if "driven by a motor", and 3) Restlessness. The most influential inattentive symptom was "Often forgetful in daily activities." When the FBATPC analysis was repeated using only the hyperactive-impulsive symptoms, the finding remained nominally significant (unadjusted $p$-value $=0.028$ ) whereas the findings was not significant when only the inattentive symptoms were used (unadjusted $\mathrm{p}$-value $=0.44$ ). After adjusting for multiple comparisons, neither finding remained significant.

\section{DISCUSSION}

Previous research used the novel analytic method, FBAT-PC, to identify an association between two SNPs in the DRD4 promoter region and a heritable quantitative phenotypes based on nine inattentive and nine hyperactive-impulsive ADHD symptoms (Lasky-Su, Biological Psychiatry in press). Using an independent population that is substantially larger in size, we evaluated 4 SNPs in DRD4 using the same methodology. Rs7124601 was found to be associated with a phenotype generated using the FBAT-PC methodology. Single nucleotide polymorphism rs7124601 was found to be associated with a phenotype generated using the FBAT-PC 
methodology. The single nucleotide polymorphism rs7124601 is located 2.27 kilobase $(\mathrm{kb})$ away from the initial finding and is located on the same haplotype block as the two previous SNPs, hCV26775267 and hCV26775266. Because these SNPs are within one haplotype block, it is possible that they are identifying the same genetic effect. Unlike the initial study, this study suggests that the phenotype is more strongly correlated with hyperactiveimpulsive symptoms, whereas the previous

\begin{tabular}{|l|l|l|l|l|}
\hline \multicolumn{1}{|l|}{ Table 2: FBAT-PC Results from the SNPs Selected using the PBAT Power Screen } \\
\hline SNPs & $\begin{array}{l}\text { Genetic } \\
\text { Model }\end{array}$ & $\begin{array}{l}\text { Number of } \\
\text { Informative Families }\end{array}$ & $\begin{array}{l}\text { Unadjusted FBAT-PC } \\
\text { p-value }\end{array}$ & $\begin{array}{l}\text { Adjusted FBAT-PC } \\
\text { p-value }\end{array}$ \\
\hline rs7124601 & Dominant & 274 & 0.478 & 0.573 \\
rs7124601 & Recessive & 330 & 0.004 & 0.021 \\
rs7124601 & Additive & 462 & 0.191 & 0.476 \\
rs3758653 & Recessive & 275 & 0.302 & 0.503 \\
rs3758653 & Dominant & 291 & 0.573 & 0.572 \\
\hline
\end{tabular}

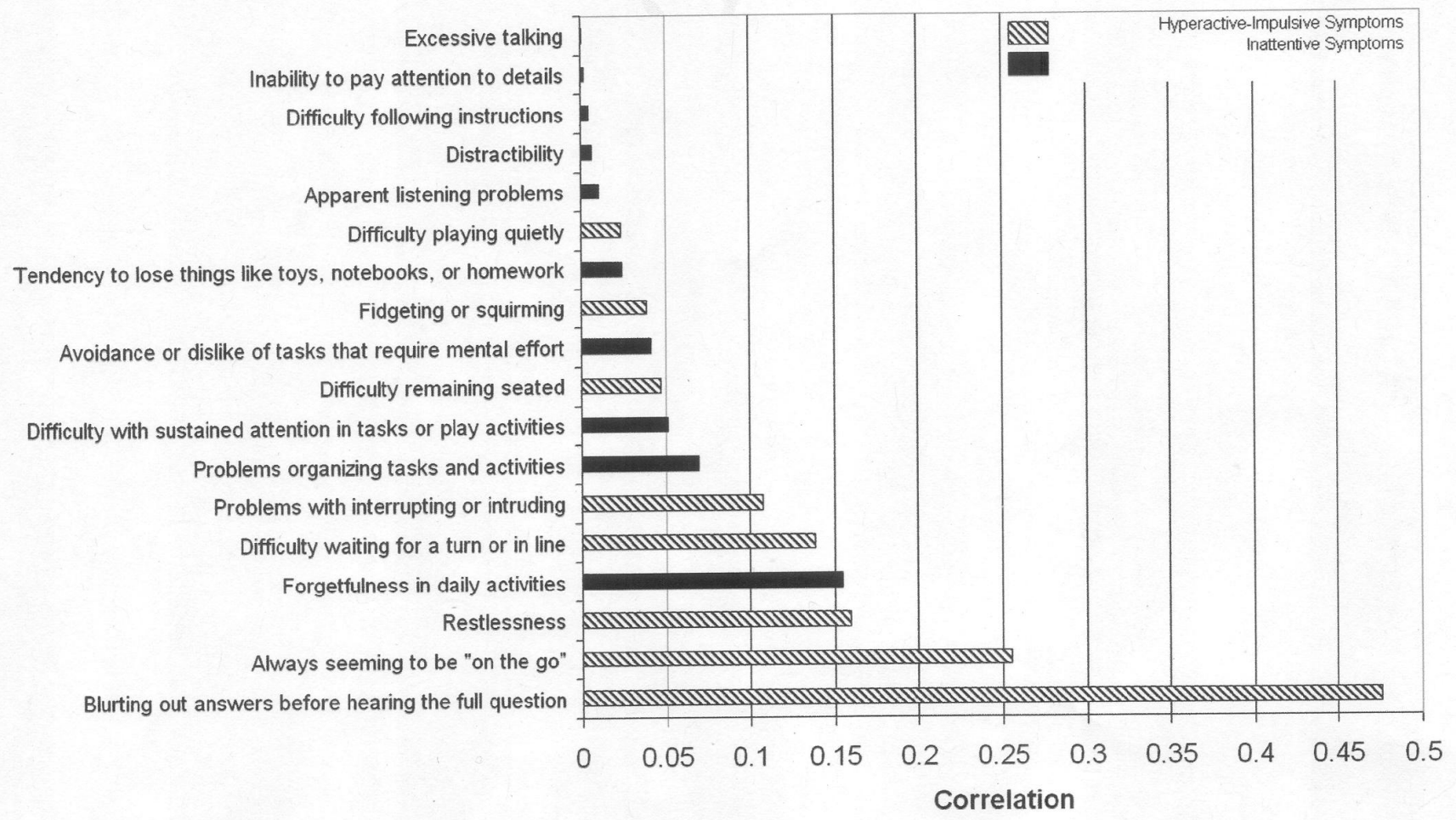

Figure 1. Correlations of ADHD symptoms with generated phenotype. ADHD, attention-deficit/hyperactivity disorder.

finding was more strongly correlated with the inattentive symptoms. It is important to note that, after multiple comparison adjustments, this finding remains significant only when both the hyperactive-impulsive and inattentive symptoms were used, suggesting that both types of symptoms are important to the overall phenotype construction. The correlation between the phenotypes generated in the first study and one generated here is modest, but significant $(r=0.19, p<0.001)$.

This attempt to replicate the initial findings is reassuring but not completely convincing. The replicate sample was sample of convenience, and therefore the SNPs that were used in the replication sample were not the same SNPs used in the 
initial study. Therefore, although the SNPs were significant in both cases, it was not an exact replication. The SNPs from both studies are on the sample haplotype block, which is reassuring, but a more ideal replication would use the original SNPs themselves. Furthermore, the significance established in the studies used different transmission models. Finally, the phenotype generated in this study was more strongly related to hyperactive-impulsive symptoms, rather than inattentive symptoms as found previously.

Although we are using a recessive model, we are not suggesting that the mode of inheritance at DRD4 is necessarily recessive. Rather, the significance of using the recessive model suggests that this is the best way to model these data using this sample. Most other studies who have evaluated the association of DRD4 and ADHD use the TDT, which does not assume a mode of transmission. Further work in larger samples would be needed to definitively clarify the mode of inheritance of the DRD4 risk variant.

The discrepancies between what is reported here and the initial findings can be explained in several ways. One possible explanation for the observed difference is differential measurement error in the collection of ADHD symptoms. In the initial study, ADHD symptoms were collected using structured interviews with the parents. The parental responses corresponded to lifetime presence of symptoms, meaning if the child ever exhibited a particular symptom, the child was recorded as having that symptom. In contrast, the Conner's scale was intended to gather current symptom information, which is likely different from lifetime symptom data. In addition, the Conner's measurements used a Likert scale with four categories whereas the initial study only has responses indicating that the symptom was absent or present. Another possible explanation for the discrepancy is that we could be identifying different genetic variations within DRD4 that influence difference characteristics of the disorder. Because rs7124601 is $160 \mathrm{bp}$ from exon 2 in DRD4, it is also possible that the association found at this SNP could be identifying effects within this exon, which would likely be different effects than what was identified in the initial paper.

There are several differences between the samples used for initial and replication studies that could also explain the observed differences in the results. The initial sample ascertained ADHD individuals based on DSM III-R and DSM IV criteria. Therefore, the individuals included in that sample could have either the inattentive, hyperactive-impulsive, or combined subtype. In contrast, the IMAGE sample recruited individuals with the combined subtype only. Since studies have found that the combined type ADHD may represent a familially independent form of disorder, the genetics underlying the IMAGE sample might be different from the initial sample.

The FBAT-PC phenotype is based on the initial estimates of heritability for each symptom. If some of the symptoms measures in one study are less accurate, decreasing the heritability estimates these symptoms, then FBAT-PC will down-weight these symptoms in its construction of the overall phenotype. Therefore, some of the differences in the relative importance of inattentive and hyperactive-impulsive symptoms between the two samples could be due to differential reliability in the symptom measurements. In the initial study, the reliability for the inattentive symptoms was 0.93 whereas the reliability coefficient for the inattentive symptoms in the IMAGE sample was only 0.45 . This change in reliability would explain why the inattentive symptoms contributed more to the overall phenotype more in the first study than in the IMAGE findings. The 
ability of FBAT-PC to either up-weight or down-weight particular symptoms is an advantage of using this methodology.

The initial report from the IMAGE study (Brookes et al., 2006) reported a nominal associations with rs9195457 and the 7repeat allele of the VNTR in exon 3 of DRD4. In the analysis, we did not analyze the 7repeat allele and we did not find rs9195457 to be significant in our analysis. The FBATPC approach is much different than the analysis strategy used in the initial paper which explains the difference in these findings. Brookes et al. (2006) did all of the analyses using a binary measure of affection status as the primary phenotype of interest whereas the FBAT-PC approach uses ordinal measures of 18 ADHD symptoms simultaneously to generate one heritable phenotype that is then used in the analysis. Because the FBAT-PC approach uses multiple phenotypes that are reduced down to a single statistical test, the FBAT-PC methodology has been shown to be more powerful than using a single binary phenotype (Lange et al. 2004a). In addition, this analysis considered multiple genetic models, whereas the initial study only considered an additive model.

This study partially replicates a previous finding that suggested that 2 SNPs in the promoter region of DRD4 were associated with a maximally heritable phenotype generated using FBAT-PC. Although the specific mechanisms by which this region of

\section{REFERENCES}

1. American Psychiatric Association (1994): Diagnostic and Statistical Manual of Mental Disorders: $4^{\text {th }}$ ed. Washington, DC: American Psychiatric Association

2. Benjamini $Y$, Hochberg $Y$ (1995): Controlling the false discovery rate: a practical and powerful approach
DRD4 affects inattentive and hyperactiveimpulsive behaviors requires additional study, this finding substantiates the previous one, suggesting that the DRD4 is associated with a phenotype generated from the 18 ADHD symptoms. Additional work is necessary to clarify which ADHD symptoms are the most influential in this association, as these findings differ from the previous ones.

\section{ACKNOWLEDGEMENTS}

The IMAGE project is a multi-site, international effort supported by $\mathrm{NIH}$ grant R01MH62873 to S.V. Faraone. Site Principal Investigators are Philip Asherson, Tobias Banaschewski, Jan Buitelaar, Richard P. Ebstein, Stephen V. Faraone, Michael Gill, Ana Miranda, Fernando Mulas, Robert D. Oades, Herbert Roeyers, Aribert Rothenberger, Joseph Sergeant, Edmund Sonuga-Barke, and Hans-Christoph Steinhausen. Senior co-investigators are Margaret Thompson, Pak Sham, Peter McGuffin, Robert Plomin, Ian Craig and Eric Taylor. Chief Investigators at each site are Rafaela Marco, Nanda Rommelse, Wai Chen, Henrik Uebel, Hanna Christiansen, Ueli Mueller, Cathelijne Buschgens, Barbara Franke, and Lamprini Psychogiou. We thank all the families who kindly participated in this research. FINANCIAL DISCLOSURES

There are no financial disclosures to report.

to multiple testing. J Roy Stat Soc 57: 289-300.

3. Brookes K, Xu X, Chen W, Zhou K, Neale B, Lowe N, et al. (2006): The analysis of 51 genes in DSM-IV combined type attention deficit hyperactivity disorder: association signals in DRD4, DAT1 and 16 other genes. Mol Psychiatry 11: 934-953 
4. Cantwell DP (1972): Psychiatric illness in the families of hyperactive children. Arch Gen Psychiatry 27: 414-417.

5. Cantwell DP (1975): Genetics of hyperactivity. J Child Psychol Psychiatry 16: 261-264.

6. Conners K (1996): Rating scales in ADHD.

7. Douglas JA, Skol AD, Boehnke M (2002): Probability of detection of genotyping errors and mutations as inheritance inconsistencies in nuclear-family data. Am J Hum Genet 70: 487-495.

8. Doyle $A E$, Faraone SV, DuPre EP, Biederman J (2001): Separating attention deficit hyperactivity disorder and learning disabilities in girls: A familial risk analysis. Am J Psychiatry 158: 1666-1672.

9. Faraone S, Biederman J, Milberger $\mathrm{S}$ (1994): An exploratory study of ADHD among second-degree relatives of ADHD children. Biol Psychiatry 35: 398-402.

10. Faraone SV, Doyle AE, Mick E, Biederman J (2001): Meta-analysis of the association between the 7repeat allele of the dopamine $d(4)$ receptor gene and attention deficit hyperactivity disorder. Am J Psychiatry 158: 1052-1057.

11. Faraone SV, Perlis RH, Doyle AE, Smoller JW, Goralnick JJ, Holmgren MA, Sklar P (2005): Molecular genetics of attention-deficit/ hyperactivity disorder. Biol Psychiatry 57: 1313-1323.

12. Freeman B, Smith $N$, Curtis C, Huckett L, Mill J, Craig IW (2003): DNA from buccal swabs recruited by mail: evaluation of storage effects on long-term stability and suitability for multiplex polymerase chain reaction genotyping. Behav Genet 33: 67-72.
13. Herbert A, Gerry NP, McQueen MB, Heid IM, Pfeufer A, Illig $T$, Wichmann HE, et al. (2006): A common genetic variant is associated with adult and childhood obesity. Science 312: 279-283.

14. Lange C, DeMeo D, Silverman EK, Weiss ST, Laird NM (2003): Using the noninformative families in family-based association tests: a powerful new testing strategy. Am J Hum Genet 73: 801-811.

15. Lange C, DeMeo D, Silverman EK, Weiss ST, Laird NM (2004a): PBAT: tools for family-based association studies. Am J Hum Genet 74: 367369.

16. Lange $C$, van Steen $K$, Andrew $T$, Lyon $\mathrm{H}$, DeMeo D, Raby B, Murphy A, et al. (2004b): A Family-Based Association Test for Repeatedly Measured Quantitative Traits Adjusting for Unknown Environmental and/or Polygenic Effects. Stat Appl Genet Mol Biol 3: 17.

17. Langley $\mathrm{K}$, Marshall $\mathrm{L}$, van den Bree $\mathrm{M}$, Thomas $\mathrm{H}$, Owen $\mathrm{M}$, O'Donovan M, Thapar A (2004): Association of the dopamine D4 receptor gene 7repeat allele with neuropsychological test performance of children with ADHD. Am J Psychiatry 161: 133-138.

18. Lasky-Su J, Faraone SV, Lange C, Biederman J, Tsuang M, Doyle AE, Smoller J, et al.: Family based association of statistically derived quantitative traits for ADHD reveal an association in DRD4 with inattentive symptoms in ADHD individuals. Biol Psychiatry in press

19. Lopez RE (1965): Hyperactivity in twins. Can Psychiatr Ass J 10: 421426.

20. Manor I, Tyano S, Eisenberg J, Bachner-Melman $R$, Kotler $M$, Ebstein RP (2002): The short DRD4 repeats confer risk to attention 
deficit hyperactivity disorder in a family-based design and impair performance on a continuous performance test (TOVA). Mol Psychiatry 7: 790-794.

21. Morrison JR, Stewart MA (1971): A family study of the hyperactive child syndrome. Biol Psychiatry 3: 189195.

22. Morrison JR, Stewart MA (1973): Evidence for polygenetic inheritance in the hyperactive child syndrome. Am J Psychiatry 130: 791-792.

23. O'Connell JR, Weeks DE (1998): PedCheck: a program for identification of genotype incompatibilities in linkage analysis. Am J Hum Genet 63: 259-266.

24. Paterson $A D$, Sunohara $G A$, Kennedy JL (1999): Dopamine D4 receptor gene: novelty or nonsense? Neuropsychopharmacology 21: 3-16.

25. Todd RD (2000): Genetics of attention deficit/hyperactivity disorder: are we ready for molecular genetic studies? Am J Med Genet 96: 241-243.

26. Welner $Z$, Welner $A$, Stewart $M$, Palkes H, Wish E (1977): A controlled study of siblings of hyperactive children. J Nerv Ment Dis 165: 110-117. 